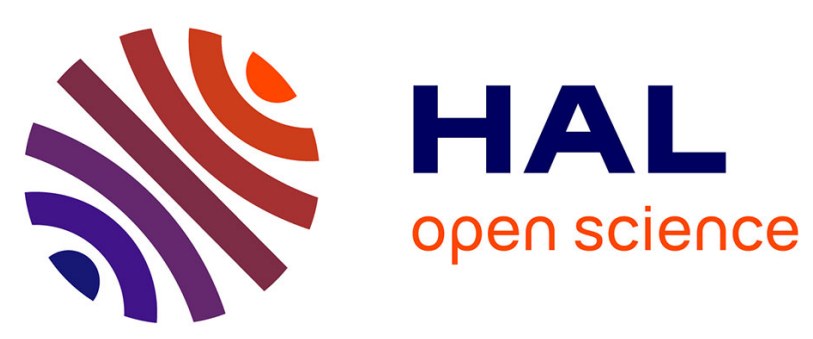

\title{
SUR UNE AMÉLIORATION APPORTÉE A LA CENTRIFUGATION SANS LE PROCÉDÉ GERBER ET SA RÉPERCUSSION SUR LES ANALYSES DU LAIT
}

M. Marc Fouassier

\section{To cite this version:}

M. Marc Fouassier. SUR UNE AMÉLIORATION APPORTÉE A LA CENTRIFUGATION SANS LE PROCÉDÉ GERBER ET SA RÉPERCUSSION SUR LES ANALYSES DU LAIT. Le Lait, 1946, 26 (259_260), pp.313-316. hal-00927928

\section{HAL Id: hal-00927928 https://hal.science/hal-00927928}

Submitted on 1 Jan 1946

HAL is a multi-disciplinary open access archive for the deposit and dissemination of scientific research documents, whether they are published or not. The documents may come from teaching and research institutions in France or abroad, or from public or private research centers.
L'archive ouverte pluridisciplinaire HAL, est destinée au dépôt et à la diffusion de documents scientifiques de niveau recherche, publiés ou non, émanant des établissements d'enseignement et de recherche français ou étrangers, des laboratoires publics ou privés. 
sécrétion, l'élaboration des lipides est davantage inhibée par l'accumulation des produits que le passage de la vitamine $\mathrm{A}^{\prime}$ du sang au au lait. Puis, bruquement le phénomène inverse se produit, et nous ne pouvons, actuellement en déterminer la cạuse.

Enfin, dans les grands intervalles; l'élaboration des lipides et le passage de la vitamine A semblent s'effectuer parallèlement.

Des difficultés tout à fait indépendantes de notre volonté (expérience effectuée en août 1944) nous ont empêchés d'effectuer un plus grand nombre de prélèvements et de dosages, les moyens de transport, l'électricité et le gaz faisant presque totalement défaut à Paris à ce moment.

Cette étude sur la composition chimique du lait en fonction de l'intervalle entre deux traites conséeutives serait à reprendre. I y aurait lieu d'étudier en détail l'élaboration des différents eenstituants du lait : des acides gras, des stérols, des phospholipides pour les lipides ; de la caséine, de l'albumine, de la globuline afin d'étudier comment la mamelle effectue la synthèse de la caséine; étude des éléments minéraux, des diastases et des vitamines enfin.

(A sunve)

\title{
SUR UNE AMÉLIORATION APPORTÉE A LA CENTRIFUQATION DANS LE PRODÉDÉ GERBER ET SA RÉPERCUSSION SUR LES ANALYSES DU LAIT (I)
}

\author{
par \\ M. MARC FOUASSIER \\ Expert chimiste près les tribunaux
}

La présente communication a pour but, non seulement de faire eonnaître une amélioration que j'ai apportée voici quelques années à la partie mécanique, autrement dit à la centrifugation qui intervient dans la méthode Gerber généralement admise dans la pratique laitière pour la détermination de la matière grasse dans le lait, mais encore de montrer par des chiffres la répereussion qu'elle peut a voir sur les résultats de l'analyse.

En effet, depuis la période froide que nous traversons, j'ai été appelé à constater des divergences sensibles et inopinées dans dés résultats fournis par des laboratoires de contrôle pour des échantillons semblables et à en rechercher les causes.

Je rappellerai tout d'abord que la méthode Gerber est basée sur la solubilisation de la caséine du lait par l'acide sulfurique en présence d'un peu d'alcool amylique. La réaction s'effectue dans une

(1) Note présentée, par G. Berirand, à l'Académie d'Agriculture de France, le 4) février 1946. 
éprouvette portant une tige graduée et dénommée butyromètré. On commence par garnir l'éprouvette de $10 \mathrm{em}^{3}$ d'acide ; on ajoute ensuite avee précaution $11 \mathrm{~cm}^{3}$ de lait puis $1 \mathrm{~cm}^{3}$ d'alcool amylique. Le butyromètré est fermé à l'aide d'un bouchon en caoutchouc, puis violemment agité, la réaction s'opère, la caséine est solubilisée et la matière grasse libérée monte dañ la tige graduée du butyromètre tenu verticalement. La température atteinte à ce moment est d'environ $85^{\circ}$ dans le liquide aeide.

Pour faciliter et activer la séparation de la matière grasse, on place les butyromètres dans une centrifugeuse spécialement aménagée pour les recevoir et dont la vitesse de rotation est d'environ 1.200 tours. La durée de la rotation doit être de trois minutes.

Il est évident, que, pendant cette opération, les butyromètres placés dans la centrifugeuse se refroidissent d'autant plus rapidement que la température ambiante est plus basse. Il importe donc, par la suite, de réchauffer ceux-ci à $65^{\circ}$ par séjour dans un bainmarie, afin de se placer dans les conditions de lecture requises par la méthode.

Afin d'éviter ce refroidissement des butyromètres au cours de la centrifugation et d'éviter également d'avoir à les réchauffer après celle-ci, j'ai imaginé de fixer immédiatement en-dessous du plateau mobilè de la centrifugeuse un plateau fixe légèrement concave pourvu d'isolateurs en porcelaine, disposés en diagonales sur toute la surface et supportant environ huit mètres de fil de résistance électrique de $4 / 10$ en ferro-nickel.

Ce dispositif, que l'on peut mettre immédiatement et facultativement, selon l'ambiance, en fonctionnement, maintient comme il est facile de le supposer, la température des butyromètres au voisinage de $65^{\circ}$. Ce mode de chauffage est continué s'il est nécessaire lorsque la centrifugeuse, étant arrêtée, on en retire un par un les butyromètres pour en faire la lecture à la température indiquée dans la technique du procédé.

Il est hors de doute que la séparation de la matière grasse est grandement facilitée par cet apport constant de caloris qui maintient sa fluidité et la rend plus apte à subir l'action de la force centrifuge.

Il en est, en effét, tout autrement lorsque les butyromètres se refroidissent d'eux-mêmes parfois aux environs de $30^{\circ}$ ou plus bas, comme cela peut arriver en période froide au cours de la centrifugation ordinaire.

Le fait de réchauffer un butyromètre à $65^{\circ}$ après la centrifugation, et j'insiste que ce point, ne permet pas d'arriver à un résultat aussi total que celui que l'on atteint en effectuant la centrifugation 
elle-même à cette température comme le permet l'amélioration dont il est question ici.

Voiei, à titre d'exemple et pour illustrer ee qui vient d'être dit, les résultats obtenus sur un même échantillon de lait standardisé à 34 grammes de provenances différentes ; j'ajoute les résultats obtenus par la méthode pondérale d'Adam, dont la précision ne saurait être mise en doute et prise ici comme contrôle, d'autre part l'exactitude du matériel utilisé a été vérifiée au préalable.

\begin{tabular}{|c|c|c|c|}
\hline Provenance & $\begin{array}{c}\text { Centrifugation } \\
\text { sans chauffage, } \\
\text { mais avec } \\
\text { réchauffage ultérieur }\end{array}$ & $\begin{array}{c}\text { Centrifugation } \\
\text { avec chauffage } \\
\text { et sans } \\
\text { réchauffage ultérieur }\end{array}$ & $\begin{array}{c}\text { Méthode pondérale } \\
\text { d'Adam }\end{array}$ \\
\hline$\ldots \ldots \ldots \ldots \ldots$ & 31,5 & 33,5 & 34,2 \\
\hline 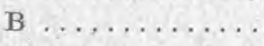 & 32,5 & 34,5 & 35,0 \\
\hline$\ldots \ldots \ldots \ldots \ldots$ & 33,0 & 35,5 & 35,8 \\
\hline$D \ldots \ldots \ldots \ldots$ & 34,0 & 36,0 & 36,5 \\
\hline $\mathbf{E} \ldots \ldots \ldots \ldots \ldots$ & $.33,0$ & 35,5 & 36,2 \\
\hline
\end{tabular}

(grammes de matière grasse par litre)

i L'examen de ces chiffres est assez concluant pour qu'il soit nutile de les longuement commenter. On remarque un écart ${ }^{c}$ onstant en moyenne supérieur à 2 grammes entre les deux modes $d_{e}$ centrifugation pratiqués. La centrifugation avec chauffage du plateau donne des chiffres très sensiblement identiques à ceux fournis par la méthode d'Adam exprimés réellement en grammes par litre de lait (la méthode Gerber donne des résultats au kilogramme conventionnellement exprimés en France en grammes par litre (Dornic et Cholet).

Ces chiffes prouvent nettement qu'il faut imputer les divergences constatées à la seule amélioration que j'ai apportée dans la centrifugation. Il ne saurait être question d'envisager celle-ci comme une modification dans la technique du procédé Gerber, elle ne orée pas évidemment de matière grasse, mais facilite sa totale extraction là où elle se trouve.

La température fayorable à une bonne séparation est fréquemment réalisée en période estivale, le chauffage du plateau de la centrifugeuse n'est pas alors rendu obligatoire ou constant. Il en est tout autrement pendant la période froide ou dans une ambiance insuffisamment chaude.

En résumé, la présente communication a pour but de faire connaître une amélioration d'ordre pratique dont un ineident a révélé 
l'importance et d'attirer l'attention sur la nécessité d'une température favorable au cours de la centrifugation des butyromètres, à mon avis trop souvent négligée malgré les répercussions qui peuvent. en découler non seulement dans des cas de contrôle ou d'expertise, mais en d'autres occasions.

Voici, en effet, un exemple : l'achat du lait à la ferme à un prix variable selon sa richesse en matière grasse conduit fréquemment à des contestations entre les cultivateurs et les acheteurs ; on ne saurait done négliger les facteurs susceptibles de les éviter. Or, la centrifugation des butyromètres dans une ambiance froide, comme dans un bon nombre de centres de campagne, conduit à un refroidissement des appareils qui ne permet pas une extraction rapide et totale. Malgré un réchauffage ultérieur, les résultats ainsi obtenus peuvent conduire à payer le lait fourni par le cultivateur au-dessous de sa valeur réelle.

J'ai cru bon de faire connaître ces faits à ceux qu'intéresse le lait ainsi que son contrôle.

\title{
PRODUCTION ET CONTROLE HYGIÉNIQUE DU LAIT DANS LES ÉTARLES URBAINES ET SURURBAINES
}

\author{
par \\ ANDRÉ LOUIS \\ Docteur-Vótérinaire sanitaire \\ (Suite)

\section{B. MESURES GÉNERALES D'HYGIÈNE}

\section{HYGIENE DE L'ALIMENTATION}

¿ Le lait est, suivant la définition légale donnée par le Congrès International de Genève pour la Répression des Fraudes, le produit. intégral de la traite totale et ininterrompue d'une femelle laitière, bien portante, bien nourrie et non surmenée.

L'alimentation a une grande importance pour la production du lait en qualité et en quantité, aussi chaque exploitant doit élever seulement le nombre de vaches qui correspond à ses possibilités.

L'établissement des rations qui doivent comprendre une quantité suffisante de principes nutritifs ne sera pas exposé ici, car il est entièrement du domaine de la zootechnie ; nous nous bornerons à indiquer :

a) Les aliments à conseiller;

b) Les aliments que l'on peut tolérer ;

c) Les aliments à rejeter.

Nous dirons enfin un mot de la boisson. 